\title{
Instant scanner device for identifying wound infection utilizing Mie scatter spectra
}

Robin E. Sweeney, Elizabeth Budiman, Jeong-Yeol Yoon

Robin E. Sweeney, Elizabeth Budiman, Jeong-Yeol Yoon, "Instant scanner device for identifying wound infection utilizing Mie scatter spectra," Proc. SPIE 10215, Advanced Environmental, Chemical, and Biological Sensing Technologies XIV, 102150U (3 May 2017); doi: 10.1117/12.2267748

SPIE Event: SPIE Commercial + Scientific Sensing and Imaging, 2017, Anaheim, California, United States 


\title{
Instant scanner device for identifying wound infection utilizing Mie scatter spectra
}

\author{
Robin. E. Sweeney ${ }^{\mathrm{a}}$, Elizabeth Budiman ${ }^{\mathrm{b}}$, Jeong-Yeol Yoon*a \\ ${ }^{a}$ Biomedical Engineering Graduate Interdisciplinary Program and Department of Biomedical \\ Engineering, The University of Arizona, Tucson, AZ, USA 85721
}

\begin{abstract}
Tissue biopsy and swab culture are the gold standards for diagnosing tissue infection; these tests require significant time, diagnostic costs, and resources. Towards earlier and specific diagnosis of infection, a non-destructive, rapid, and mobile detection device is described to distinguish bacterial species via light scatter spectra from the surface of an infected tissue, reagent-free. Porcine skin and human cadaveric skin models of wound infection were used with a $650 \mathrm{~nm}$ LED and an angular photodiode array to detect bacterial infections on the tissue surface, which can easily be translated to a typical CMOS array or smartphone. Tissue samples were inoculated with Escherichia coli, Salmonella Typhimurium, or Staphylococcus aureus and backscatter was collected from $100^{\circ}$ to $170^{\circ}$ in $10^{\circ}$ increments; each bacterial species resulted in unique Mie scatter spectra. Distinct Mie scatter spectra were obtained from epidermis (intact skin model) and dermis (wound model) samples, as well as from porcine and human cadaveric skin samples. Interactions between bacterial colonies and lipid particles within dermis samples generated a characteristic Mie scatter spectrum, while the lipid itself did not contribute to such characteristic spectrum as corroborated with body lotion experiments. The designed angular photodiode array is able to immediately and non-destructively detect tissue bacterial infection and identify the species of infection within three seconds, which could greatly improve point of care diagnostics and antibiotic treatments.
\end{abstract}

Keywords: Diabetic foot ulcer; Light scatter; Escherichia coli, Salmonella Typhimurium; Staphylococcus aureus

\section{INTRODUCTION}

Anytime the surface of the skin is broken, there is risk of infection. Generally, if not noticed and treated quickly, infection delays healing time and increases risk of complications, especially among diabetic, elderly, or traumatic burn victim patients. Early diagnosis of an infection leads to early treatment and, therefore, better prognosis for the patient, decreasing the risk of wound infection becoming limb or life threatening ${ }^{1,2,3}$. It is estimated that care for chronic wounds carries an economic burden of \$6-\$15 billion annually in the United States (US) alone; infection on a chronic wound significantly increases the cost of care ${ }^{4}$, and some studies even suggest that the cost in the US is truly as high at $\$ 25$ billion annually ${ }^{5}$.

Antibiotic treatment differs based on the bacterial species infecting a wound and severity of the wound ${ }^{2}$. Currently, wound infection is initially suspected based on visual changes to a wound, such as redness caused by inflammatory responses of a patient. However, these visual changes are difficult to distinguish in a chronic wound, as severe inflammation exists independent of bacterial infection ${ }^{2,6}$. To diagnose infection, the wound must be biopsied or swabbed followed by culture and subsequent stain of these samples (e.g., Gram stain), which typically takes a day or two; diagnosis is therefore, an invasive, painful, and time consuming process that can only be carried out in an expensive laboratory environment ${ }^{1,3,6,7}$. Further, studies have indicated that while pain is a good indicator of infection, lack of pain is not a good indicator of lack of infection, making early signs of infection difficult to distinguish ${ }^{7}$. During the lengthy procedure to specifically diagnose wound infection, patients are often prescribed broad-spectrum antibiotics, which are known to contribute to antibiotic resistance. Early and specific diagnosis of wound infection prevents unnecessary use of broad-spectrum antibiotics and can prevent complications in treatment due to polymicrobial colonization of a wound ${ }^{2}$.

*jyyoon@email.arizona.edu; phone 1520 621-3587; biosensors.abe.arizona.edu

Advanced Environmental, Chemical, and Biological Sensing Technologies XIV, edited by

Tuan Vo-Dinh, Robert A. Lieberman, Proc. of SPIE Vol. 10215, 102150U · (c) 2017 SPIE

CCC code: $0277-786 \mathrm{X} / 17 / \$ 18 \cdot$ doi: $10.1117 / 12.2267748$ 
Risk of wound infection is high across a diverse patient population. Compromised microvasculature causes poor immune response and delayed healing in diabetic patients, leading to high rates of diabetic foot ulcer (DFU) infections, most commonly Staphylococcus aureus and Escherichia coli ${ }^{2}$. In the US alone, 29.1 million people suffer from diabetes, of which $15 \%$ suffer from chronic DFU; DFU is the primary reason for non-traumatic limb amputations in the US with an estimated $15-20 \%$ of DFU leading to lower extremity amputations ${ }^{8,}{ }^{9}$. Chronic infection of DFU and related complications increase the instance of amputation to $28-96 \%{ }^{10,11}$. Decline of the innate and adaptive immune systems in elderly population results in elderly patients experiencing delayed wound healing and increased risk for infection ${ }^{12}$. There is obvious concern that problems associated with age will only multiply as the population ages, with risk of wound infection being no exception. Gram-negative infections, namely E. coli, predominate in burn wounds and are a major determinant of morbidity and mortality; burn wound infections often result in sepsis, which ultimately causes $75 \%$ of post-burn deaths ${ }^{13,14}$. In cases where burns are sustained, inflammation and immune suppression are associated with the initial injury, meaning diagnosis is increasingly more challenging in these patients ${ }^{13}$. It is estimated that $2-5 \%$ of surgeries result in surgical site infection (SSI), a major complication that is key in morbidity, mortality, and need for corrective surgery ${ }^{15,16}$. In surgical patients, as well as lacerations in general, location of the wound has been shown to correlate to rate of infection, so monitoring incisions and lacerations at susceptible location closely could improve prognosis and decrease the economic burden of infection ${ }^{16,17}$. It is clear that many diverse populations of patients are in need of a diagnostic method for wound infection that is significantly faster and reliable.

A faster and more affordable approach to infection diagnosis is necessary to reduce health care costs, decrease time to treatment, and increase efficacy of treatment across a wide variety of populations. A mobile diagnostic device is essential to warn patients of infection early, despite visual and physical markers of infection being difficult to distinguish. Mobile monitoring of a wound would allow early notification of infection to a patient and a physician to allow early treatment and, therefore, improved efficacy of treatment, prognosis, and quality of life. Rapid optical analysis of a wound surface eliminates the need for any reagents, lengthy assay times, tedious sample handling, and the need for access to skilled technicians in a laboratory.

In this work, we utilize Mie scatter spectra to directly detect wound infection from animal and human skin models. Mie scatter is dependent on particle size, morphology, refractive index, and concentration. Changes in these factors due to bacterial growth and interactions with tissue components, especially lipids, result in changes in scatter patterns across a variety of angles ${ }^{18}$. Our device takes advantage of changes in Mie scatter patterns based on these factors. In this work, we utilize Mie scatter spectra collected from various wound models, of both porcine and human skin, to rapidly detect bacterial infections on tissue surfaces and determine the bacterial species responsible for the infection. Unlike other biosensing techniques, such as the use of Mie scatter to detect bacterial presence through particle immunoagglutination ${ }^{19}$, ${ }^{20}$, this method does not require sample processing, additional reagents, or bioreceptor use, which eliminates time and labor for lengthy reagent and sample preparation.

Several studies have reported the use of Mie scatter imaging to identify bacteria grown on agar plates, but not directly from a wound or tissue. Our technique uses back scatter spectra from a tissue surface, unlike Mie scatter imaging which uses forward scatter images through colonies to identify patterns ${ }^{21}$. Mie scatter imaging systems, often referred to as BARDOT systems, require bacteria to be cultured on before identification of bacterial species, as BARDOT systems are used to identify fully developed cultures on an optically transparent substrate $22,23,24$. The system designed here does not require the additional steps of culturing a wound sample to determine the presence or cause of bacterial infection. The technique described in this work differs in that rather than detecting forward scatter through agar, backscatter is detected from a tissue surface, meaning that culture of a suspected bacterial infection is not necessary, eliminating significant sample processing time and expenses.

Towards this goal, a custom stage was developed to monitor Mie backscatter from a tissue surface using a $650 \mathrm{~nm}$ LED. Mie scatter is detected at $10^{\circ}$ increments from $100^{\circ}$ to $170^{\circ}$. Our novel device is capable of detecting infection on a tissue surface and the bacterial species responsible within three seconds, without physically contacting a sample, and without using any reagents such as stain, antibody, or nucleic acid. Scale up of our device would allow a patient to simply scan a wound to determine if an infection is present, and if so, the species of the infection.

Our goal was to develop a miniature prototype of such a device for rapid and mobile diagnosis of an infected wound, as well as to determine the ability to differentiate species of bacterial infection. 


\section{MATERIALS AND METHODS}

\subsection{Study design}

Study groups in this controlled laboratory experiment include Escherichia coli, Salmonella Typhimurium, Staphylococcus aureus, and a control of sterile LB-Miller medium on four surfaces; LB-Miller agar, porcine dermis, porcine epidermis, human cadaveric epidermis. Mie scatter spectra were collected using a custom angular photodiode array at $10^{\circ}$ increments from $100^{\circ}$ to $170^{\circ}$ (details of this device are discussed in section 2.5). Each sample was analyzed at three separate locations for a representative collection of scatter from an entire surface. Samples were randomly assigned an inoculum and researchers were not blinded.

\subsection{Bacteria solutions}

Escherichia coli K12 (Sigma-Aldrich, St. Louis, MO, USA), Salmonella Typhimurium (ZeptoMetrix, Buffalo, NY, USA), and Staphylococcus aureus (ZeptoMetrix, Buffalo, NY, USA) were all individually cultured in lysogeny broth (LB) Miller's formula (Molecular Biologicals International Inc, Irvine, CA, USA) at $37^{\circ} \mathrm{C}$ for 8 hours. Cultures were plated on LB-Miller agar plates (BIO5 Media Facility, Tucson, AZ, USA) by uniformly spreading $100 \mu \mathrm{L}$ of a bacteria culture across the surface. Cultured agar was cut to approximately $1.5 \mathrm{~cm}$ by $5 \mathrm{~cm}$ and placed on microscope slides with care taken to avoid disrupting the surface of the agar. Pure bacteria cultures on agar were analyzed using the photodiode array described below.

\subsection{Tissue sample preparation: porcine skin}

Porcine skin (University of Arizona Food Products and Safety Laboratory, Tucson, AZ, USA) was acquired immediately following slaughter. Hair was shaved from the skin surface, samples were randomly divided into two experimental groups; exposed porcine epidermis and porcine dermis. The porcine epidermis experimental group was not further processed. The porcine dermis experimental group was further processed by mechanically dissecting the epidermis, exposing the dermis of the skin (Fig. 2). Samples in both groups were cut into approximately $1.5 \mathrm{~cm}$ by $5 \mathrm{~cm}$ rectangles and inoculated with $150 \mu \mathrm{L}$ of either E. coli, S. Typhimurium, S. aureus, or sterile LB-Miller medium, all of which were spread evenly across the surface of the skin with an inoculating needle. Samples were sealed with Parafilm M (Bemis Flexible Packaging, Oshkosh, WI, USA) and incubated for 8 hours to allow bacterial growth on the sample.

Rather than bacterial inoculation, alternative porcine dermis samples were coated with body lotion (St. Ives Skin Renewing Collagen Elastin, Unilever, Englewood Cliffs, NJ, USA), a common skin contaminant. Preparation and analysis of lotion coated skin samples was identical to that of bacteria inoculated samples, with the exception of inoculum.

\subsection{Tissue sample preparation: human cadaveric skin}

Human cadaveric skin was acquired from the ankle region. Cadaveric skin samples were prepared into identical samples as porcine skin. The epidermis remained intact for all samples due to frailty of the skin. Samples were allowed to rest for 15 minutes at room temperature following inoculation of $150 \mu \mathrm{L}$ of each bacteria culture before analysis.

\subsection{Mie scatter detector}

Directly following incubation of tissues, samples were placed on a standard microscope slide with care taken to not disrupt the surface of the skin. A 3D printed stage held the microscope slide in a fitted channel to be analyzed by a custom photodiode array. A $650 \mathrm{~nm}$ red LED light source was placed perpendicular to the surface of the sample with PIN photodiodes detecting scatter off of the tissue sample at $10^{\circ}$ increments from $100^{\circ}$ to $170^{\circ}$ relative to the tissue surface (Fig. 1a). All photodiodes were used in photovoltaic mode and connected to LM324 quad op-amps to amplify current outputs. Amplified signals were sent to the analog inputs of an Arduino Mega 2560 microcontroller, providing an angular spectrum. Each tissue sample was analyzed at three different locations. Photodiode readings were collected every $250 \mathrm{~ms}$ and averaged over three seconds (12 data points per each angle). For each sample, photodiode readings were averaged at each angle, and standard error was calculated over three different tissue locations of multiple samples for the purpose of error bars.

As a control experiment, tissue samples were stamped on LB-Miller agar (BIO5 Media Facility) by inverting the tissue sample, pressing the inoculated surface against the surface of the agar briefly, and incubating the agar plates for 8 hours at $37^{\circ} \mathrm{C}$. Agar stamps were cut the stamped portion and placing it on a microscope slide with care taken to avoid disrupting the surface of the agar followed by analysis using the designed array. 

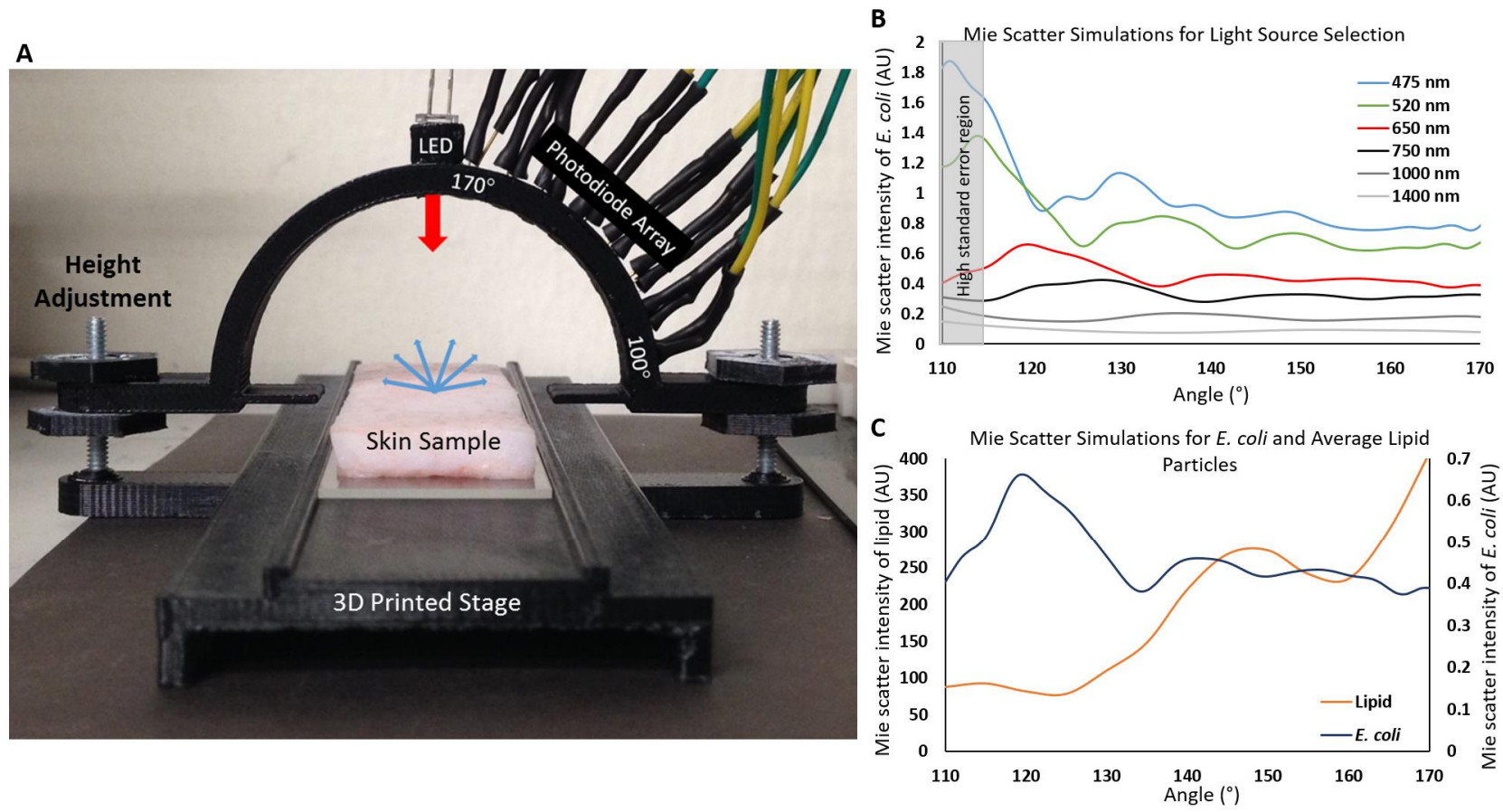

Figure 1. Device design and expected results. (A) Designed device. Inoculated samples were incubated at $37^{\circ} \mathrm{C}$ for 8 hours and analysed using the angular photodiode array shown. The array is height adjustable to ensure analysis at the surface of each individual sample. A $650 \mathrm{~nm}$ LED perpendicular to the tissue surface illuminates the sample and Mie scatter spectra are detected at increments of $10^{\circ}$ from $100^{\circ}$ to $170^{\circ}$. (B) Justification of red LED use. Simulations of Mie scatter spectra for E. coli with a variety of light source wavelengths. Based on these simulations a $650 \mathrm{~nm}$ red LED was chosen as the light source for the designed device. (C) Mie scatter simulation for individual E. coli bacterium and individual lipid particles using MiePlot v4.2.11. Distinct differences are seen between expected Mie scatter spectra from individual bacterium and those from lipid particles.

\subsection{Mie scatter simulation}

Mie scatter simulations were carried out using MiePlot v4.2.11. For lipids, the following parameters were used ${ }^{18}$ : particle radius $=10 \mu \mathrm{m}$, refractive index $=1.46$, refractive index of medium (water) $=1.33$, wavelength $=650 \mathrm{~nm}$, and size distribution was assumed to follow a normal distribution with $10 \%$ standard deviation. For individual E. coli, the following parameters were used ${ }^{18,19}$ : particle radius $=2.5 \mu \mathrm{m}$ (hydrodynamic dimensions of $5 \mu \mathrm{m} \times 1 \mu \mathrm{m}$ ), refractive index $=1.40$, refractive index of medium (water) $=1.33$, wavelength $=650 \mathrm{~nm}$, and size distribution was assumed to follow a normal distribution with $10 \%$ standard deviation. To determine the appropriate light source for the device, simulations were conducted based on E. coli, varying only wavelength of the light source from $475 \mathrm{~nm}$ to $1400 \mathrm{~nm}$.

\section{RESULTS}

\subsection{Device design}

Final device design is shown in Figure 1a. A semi-circle angular array was 3D printed with insertion points for an LED, perpendicular to the tissue surface, and PIN photodiodes, at $10^{\circ}$ increments from $100^{\circ}$ to $170^{\circ}$. The array was attached to a 3D stage, designed to fit a standard microscope slide, in a manner that would allow adjustment of array height relative to the stage. Array height was adjusted prior to each measurement, so the array was level with the surface of a tissue sample.

\subsection{Light source selection}

Following Mie scatter simulations (MiePlot v4.2.11) a $650 \mathrm{~nm}$ red LED was used as a sole light source. Mie scatter simulations were performed for $E$. coli (radius $=2.5 \mu \mathrm{m}$, normal size distribution with $10 \%$ standard deviation, refractive index $=1.40$, refractive index of medium $=1.33$ ) at wavelengths of $475 \mathrm{~nm}, 520 \mathrm{~nm}, 650 \mathrm{~nm}, 750 \mathrm{~nm}, 1000 \mathrm{~nm}$, and $1400 \mathrm{~nm}$ (Fig. 1b). Wavelengths in the ultraviolet (UV) range were not considered due to their bactericidal capability and dangers of UV exposure to human skin and wound. Side scatter results in large variability in data collected at $100^{\circ}$ 
and $110^{\circ}$, due to the presence of hair follicles and imperfections in the topography of tissue samples. Major peaks of $E$. coli scattering were identified for the above wavelengths of light source through Mie scattering simulations. Light sources at $475 \mathrm{~nm}$ and $520 \mathrm{~nm}$ were not considered due to the major peaks of $110.9^{\circ}$ and $111.1^{\circ}$, respectively. NIR wavelengths $(750 \mathrm{~nm}, 1000 \mathrm{~nm}$, and $1400 \mathrm{~nm})$ were also ruled out due to low intensity major peaks and that the eventual goal of translation to smartphone would require an additional NIR light source. A $650 \mathrm{~nm}$ LED light source was selected based on a high intensity major peak at $119.9^{\circ}$, which avoids variability at low angles of detection, the designed device is able to detect this peak with a detector at $120^{\circ}$. Figure 1c shows expected Mie scatter patterns for E. coli at this wavelength as well as for lipid particles, which are critical in the dermis layer of tissue.

\subsection{Sample preparation}

Porcine skin was collected immediately following sacrifice and human cadaveric skin was collected following a period of deep freeze. Hair was shaved from the skin surface to successfully expose intact epidermis of porcine skin and human cadaveric skin. Mechanical dissection of the epidermis exposed the dermis of porcine skin, successful dissection was validated via light microscopy. Similar mechanical dissection of the epidermis was not possible with human cadaveric skin, limiting the human cadaveric skin experiments to epidermal infection case. Procedure and exposure of epidermis and dermis layers are shown in Figure 2 with the expected interactions of each tissue layer with inoculated bacteria.

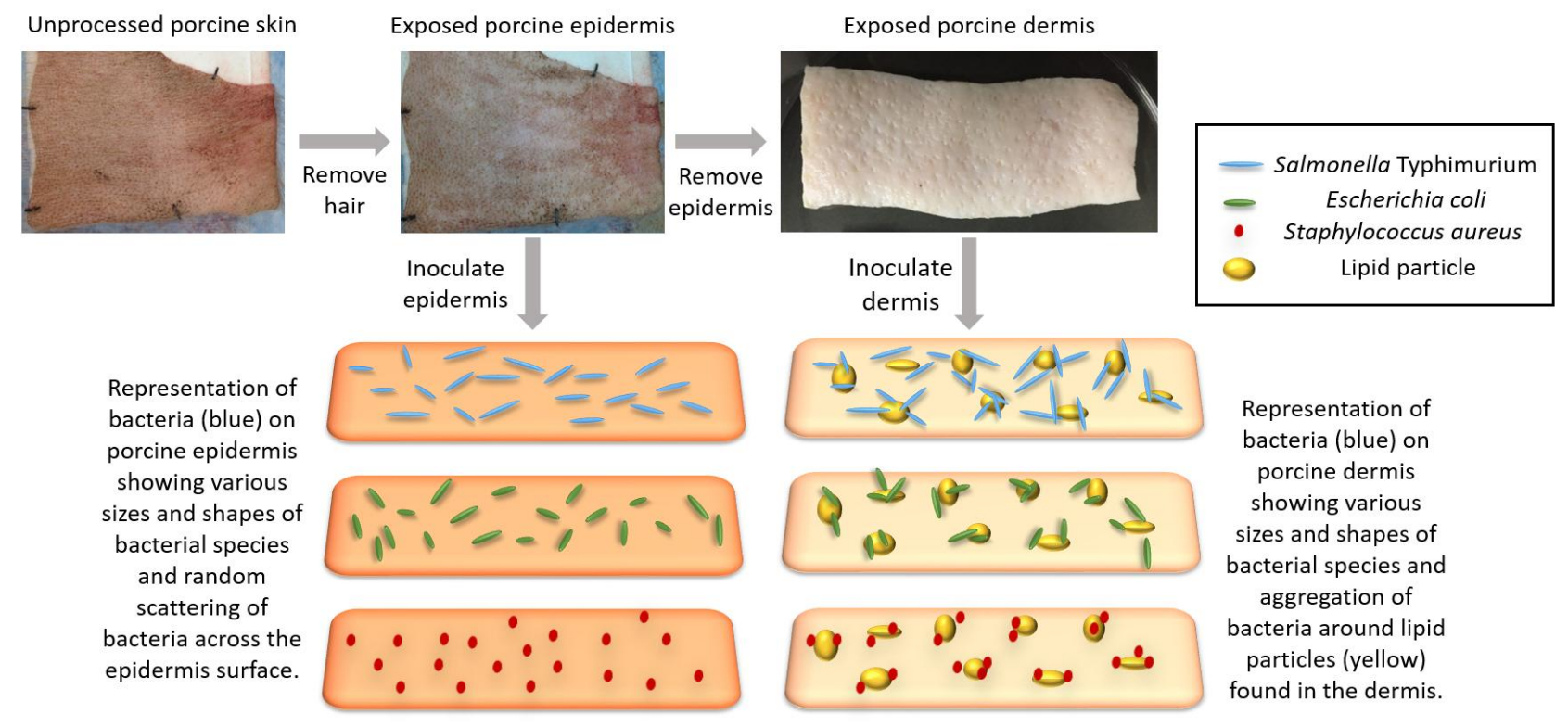

Figure 2. Skin treatment. Hair was shaved off of porcine skin, exposing the epidermis. Exposed epidermis was either divided into experimental groups and inoculated, or mechanically dissected away, exposing the dermis. Samples with dermis exposed were divided into experimental groups and inoculated. Schematic diagrams show the expected distribution of bacteria on each tissue surface as well as differences in size and shape between bacterial species tested. The epidermis layer does not contain exposed lipid particles, so bacteria will grow even across the surface. The dermis layer does contain exposed lipid particles, it is know that bacteria aggregate around these particles, forming pseudo-colonies.

\subsection{Bacteria on agar}

As controls, bacteria grown on agar plates were tested using the designed photodiode array shown in Figure 1a. Significant differences were detected between Mie scatter spectra of three bacterial species grown on LB-Miller agar despite no obvious visual differences between cultures on agar (Fig. 3). A single peak is observed at $120^{\circ}$ for all bacterial species, but differences in intensity are obvious between the three species analyzed, despite identical concentrations of each culture $\left(10^{8} \mathrm{CFU} / \mathrm{mL}\right.$, confirmed by colony counting). Obtained data correlates with the expected $120^{\circ}$ peak based on Mie scatter simulations for E. coli (Fig. 1c). 


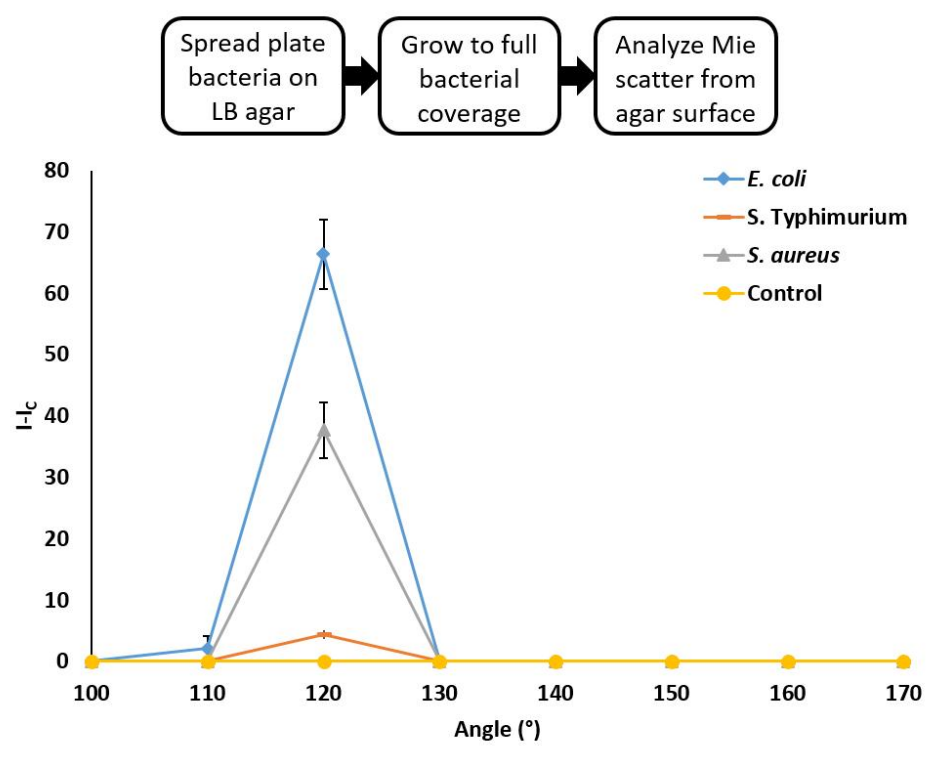

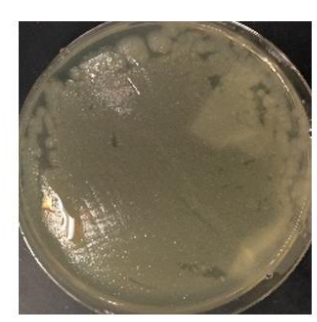

E. coli

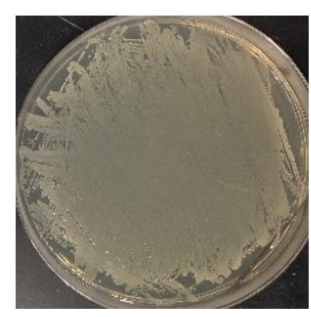

S. aureus

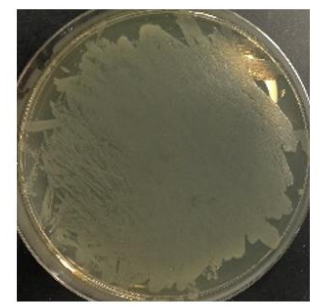

S. Typhimurium

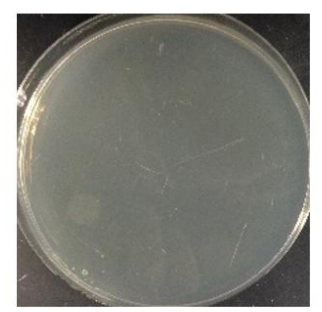

Control

Figure 3. Mie scatter spectra from agar surface. Pure bacteria cultures were cultured on LB-Miller agar until the surface of the agar was fully covered with colonies. Mie scatter from the agar surface was analyzed exactly as in the case of tissue samples. Data from bacteria on agar is normalized to a control of sterile LB-Miller agar by subtracting the signal intensity of the control sample $\left(\mathrm{I}_{\mathrm{C}}\right)$ from the signal intensity of the bacteria sample (I). Since agar is quite transparent, $\mathrm{I}_{\mathrm{C}}$ is frequently zero, thus the normalization $\left(\mathrm{I}-\mathrm{I}_{\mathrm{C}}\right)$ is different from those used in Figs. 4 and $6\left(\mathrm{I} / \mathrm{I}_{\mathrm{C}}\right)$. Error bars represent standard error.

\subsection{Bacteria on porcine epidermis}

Bacteria grown on porcine epidermis resulted in a similar trend to that of bacteria grown on agar, with the exception that the major peak is shifted to $130^{\circ}$ and normalization to porcine epidermis inoculated with sterile LB-Miller medium inverts to a minimum (Fig. 4a). Peak inversion can be explained by the reflective nature of porcine epidermis samples, presumably because the porcine samples were acquired and processed right after subjects were sacrificed (i.e. within hours). Light scatter from bacterial colonies and lipid particles negatively contributed to such reflection, thus the normalized spectra were inverted. No such inversion was found for porcine dermis (no reflection - essentially exposed tissue), human cadaveric epidermis (aged for years), or agar (optically transparent). Interactions with compounds in the skin are believed to cause the shift in angle of the peak between agar and porcine epidermis. Though the peak shifts to $130^{\circ}$, this is within the range of the expected peak based on Mie scatter simulations shown in Figure 1c.

\subsection{Bacteria on porcine dermis}

Bacteria were grown on porcine dermis, simulating the infection on an actual wound. Each bacterial species maintains a unique and distinguishable trend (Fig. 4b), despite no obvious visual differences between inoculated samples, but that these trends are more complex than the single major peak of the pure cultures and porcine epidermis. All spectra collected from inoculated porcine dermis are normalized to that of porcine dermis inoculated with sterile LB-Miller medium. Mie scatter simulations result in expected pattern difference between lipid particles and individual bacterium (Fig. 1c). The lipid particles show negligible scattering at low scatter angles, a rapid increase over $130^{\circ}-150^{\circ}$, and a saturation at high scatter angles, while $E$. coli particles show a single peak around $120^{\circ}$. Lipid particles are present in the dermis layer; bacteria have been shown, previously, to aggregate around these lipid particles, as shown in Figure 2, resulting in a complex trend unique to the infected dermis. The spectra of bacteria on porcine dermis seem to be a combination of both lipid and E. coli particles - the rapid increase is shifted to low scatter angle $\left(100^{\circ}-120^{\circ}\right)$ presumably due to the $120^{\circ}$ peak of E. coli. It should also be noted that Staphylococcus aureus spectra are substantially different from those of E. coli and Salmonella Typhimurium, on both epidermis and dermis (Fig. 4). Reproducibility was shown through repeated experiments on porcine dermis from the same individual. 


\section{A Spectra from Inoculated Porcine Epidermis}
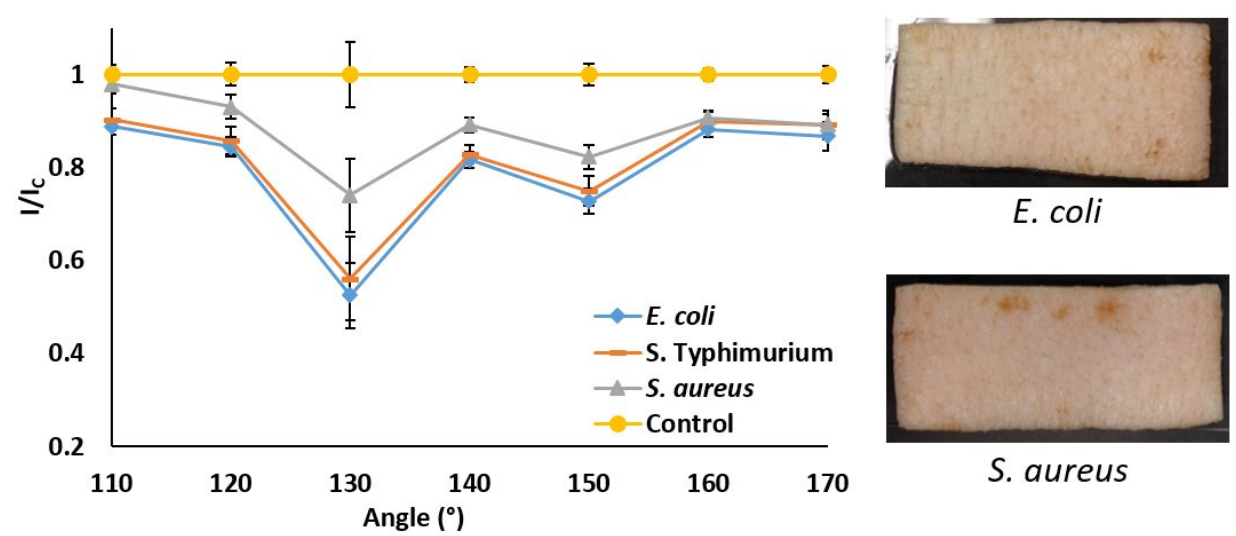

B Spectra from Inoculated Porcine Dermis

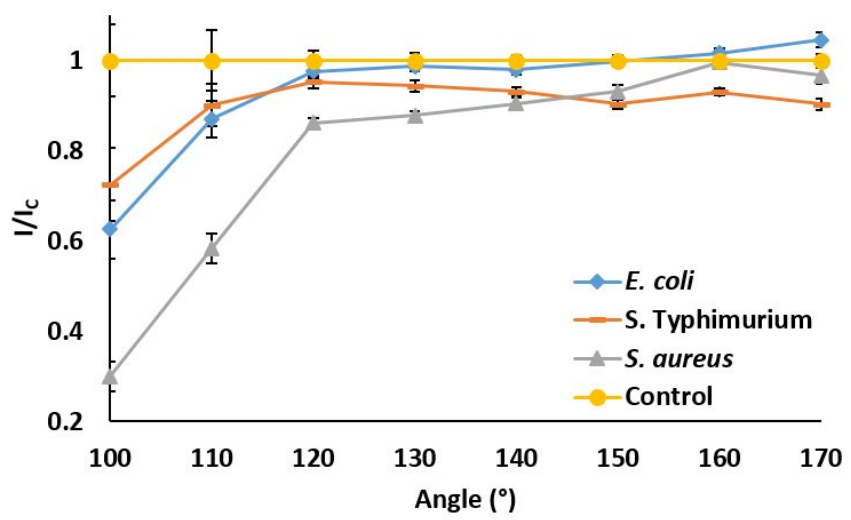

E. coli

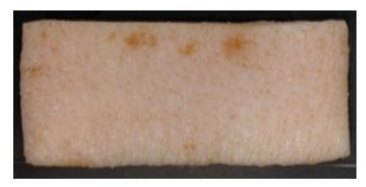

S. aureus

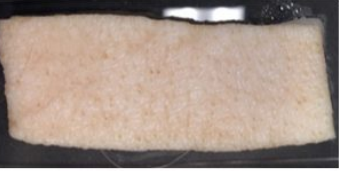

S. Typhimurium

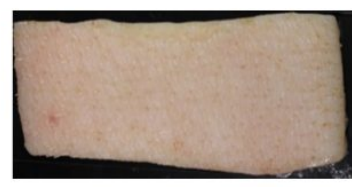

Control

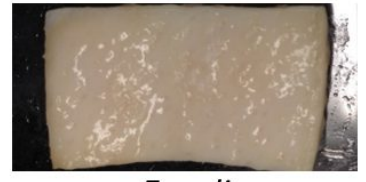

E. coli

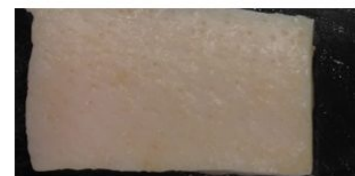

S. Typhimurium

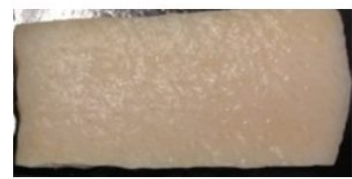

S. aureus

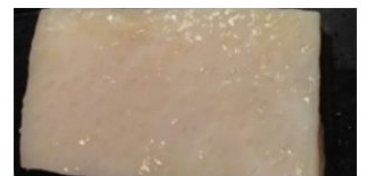

Control

Figure 4. Inoculated porcine skin samples. (A) Bacterial cultures on porcine epidermis. Normalized scatter intensities of $E$. coli, $S$. Typhimurium, and $S$. aureus on porcine epidermis, showing similar spectra to those on agar, while being inverted. Side scatter results in large variability in data collected from $100^{\circ}$ to $110^{\circ}$ (thus not shown), due to the presence of hair follicles and imperfections in the topography of porcine epidermis. (B) Bacterial cultures on porcine dermis. Normalized scatter intensities of $E$. coli, $S$. Typhimurium, and $S$. aureus on porcine dermis. Both A and B show significant differences in spectra among three bacterial species, especially between gram negative ( $E$. coli and $S$. Typhimurium) and gram positive ( $S$. aureus). Images shown to the right of each show no clear, visual differences between inoculations, while the designed device is capable of detecting such differences. Signal intensities of bacterial inoculated porcine skin samples (I) are normalized to sterile LB-Miller medium inoculated control skin samples $\left(\mathrm{I}_{\mathrm{C}}\right)$ for both graphs. All data points are the averages of at least three different experiments. Error bars represent standard error.

\subsection{Skin contaminants on porcine dermis}

Exposed porcine dermis was coated in body lotion, a common lipid based skin contaminant. No significant difference between control samples and lotion-coated samples was detected (Fig. 5).

\subsection{Bacteria on human cadaveric epidermis}

Human cadaveric skin inoculated with bacteria resulted in major peaks at $130^{\circ}$ and $170^{\circ}$ (Fig. 6). The major peaks observed on human cadaveric epidermis are highly similar those observed on porcine epidermis, on agar plates, and through Mie scatter simulations. The major peak at $130^{\circ}$ is remarkably similar to data in Figure $4 \mathrm{a}$ collected from porcine epidermis, while the peak at $170^{\circ}$ is a unique characteristics for human skin. 

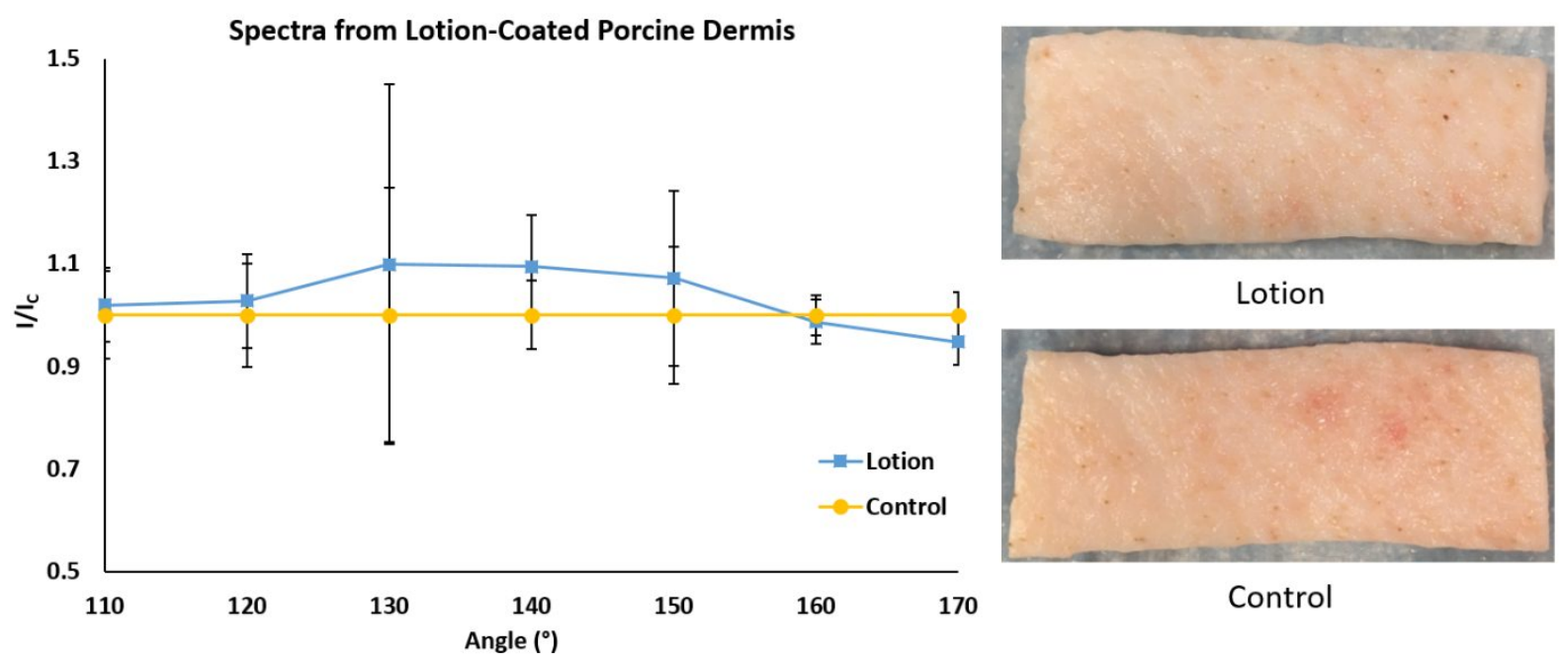

Control

Figure 5. Lotion coated porcine dermis. No significant differences are seen between lotion coated skin samples and control skin samples, showing that common skin contaminants are not detected by the designed system. All data points are the averages of at least three different experiments. Error bars represent standard error.

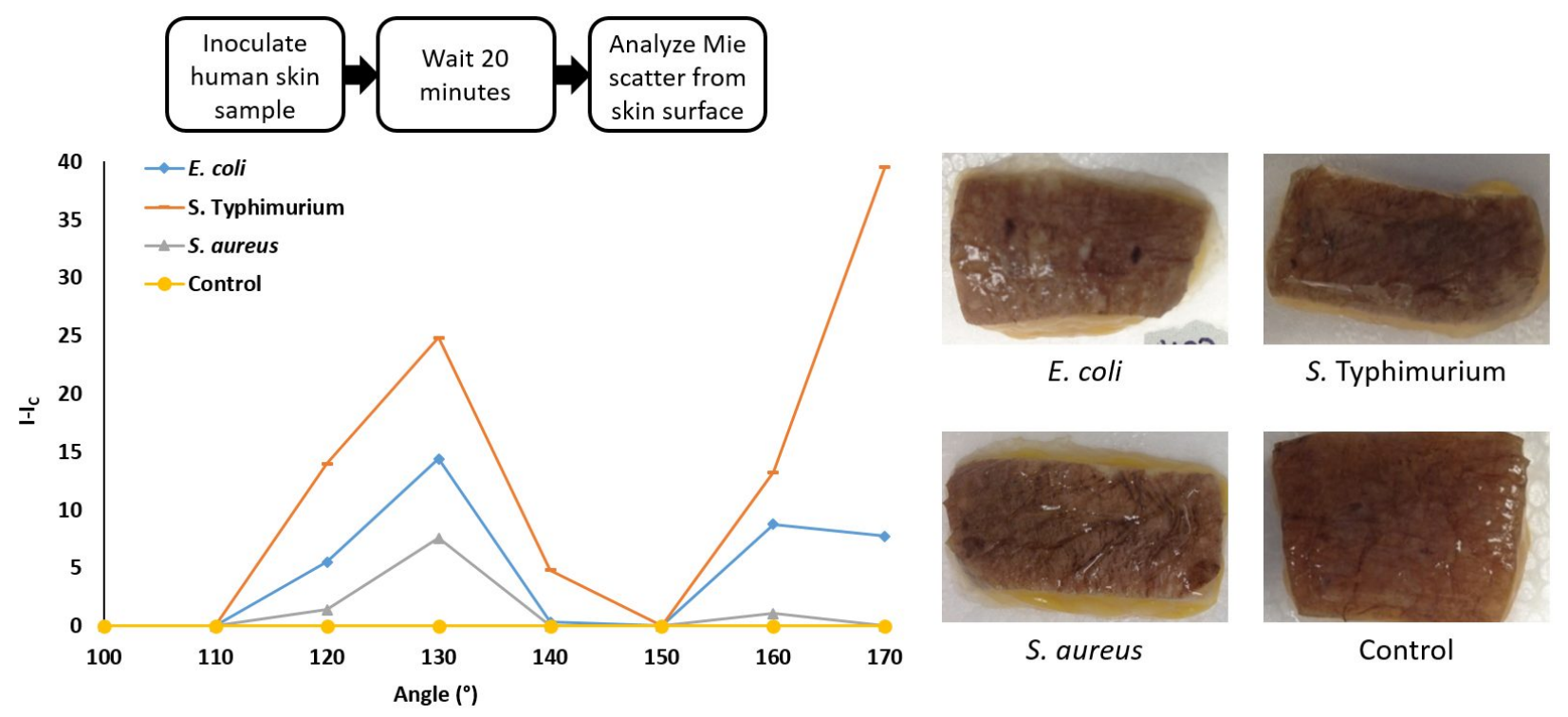

Figure 6. Mie scatter spectra on human cadaveric skin. Normalized scatter intensities of $E$. coli, $S$. Typhimurium, and $S$. aureus on human cadaveric skin. Major peaks are seen at $130^{\circ}$ and $170^{\circ}$. Similarly to the porcine epidermis, the major peak at $130^{\circ}$ shows distinct differences between the three different bacterial species. Signal intensities of bacteria inoculation human cadaveric skin samples (I) were normalized to sterile LB-Miller inoculated control human cadaveric skin samples $\left(\mathrm{I}_{\mathrm{C}}\right)$. All data points are the average of three different experiments. Error bars represent standard deviation.

\section{DISCUSSION}

\subsection{Bacteria-lipid aggregates}

Our lab has previously demonstrated that E. coli aggregate around lipid particles in ground beef and this aggregation changes Mie scatter patterns in a concentration dependent manner ${ }^{18}$. It is believed that this aggregation is also occurring when bacteria are inoculated on the surface of porcine dermis used in this study, while the shape and morphology of such aggregation is quite different from the case of ground beef (Fig. 2). In wound infection, bacteria grow within 
damaged/exposed dermis for a long period of time, while our previous work detects the microbial spoilage or contamination that happens for quite a short period of time. The resulting Mie scatter spectra from bacteria on porcine dermis are therefore quite different from those from ground beef. The Mie scatter spectra on the epidermal surface of both porcine and human cadaveric skin are more similar to those observed from bacteria on agar. The dermis provides lipid "particles" for the bacteria to aggregate around, while agar and epidermis do not provide substantial amounts of lipid "particles" (Fig. 2). The device designed can distinguish between bacteria on either dermis or epidermis, so it is able to successfully detect differences in the growth patterns of the bacteria on various wound surfaces.

\subsection{Identification of specific bacterial species}

Cultures of individual bacterial species were inoculated on porcine and human cadaveric skin samples to mimic infection. Sterilized LB-Miller medium was used, to directly compare to the bacteria cultures grown in the same broth. Porcine dermis was exposed to mimic the exposure of the dermis in a wound environment. All bacteria cultures were grown to maximum concentration $\left(10^{8} \mathrm{CFU} / \mathrm{mL}\right)$, where this concentration was determined through serial dilution and plate counts. A unique trend can be detected between each bacterial species used in this study, showing that rapid identification based on Mie scatter is possible in wound infection. The trends of gram-negative bacillus bacteria (E. coli and $S$. Typhimurium) are noticeably more similar than that of gram-positive cocci bacteria (S. aureus). The size and shape of individual bacterium determine Mie scatter spectra, so it would be expected that bacteria similar in these categories have similar trends. Notable differences between gram-negative bacilli bacterial species and gram-positive cocci bacterial species is critical in a robust wound infection detection system. S. aureus, Pseudomonas aeruginosa, and $E$. coli are some of the most common pathogens in wound infection, and these bacteria differ in size, shape, and Gram stain.

\subsection{Verification of bacterial growth after inoculation}

To verify that the inoculated bacteria was in fact growing on the tissue surface following inoculation, culture, and analysis, the surface of all samples was stamped on LB-Miller agar and cultured. Bacteria grown from stamping the surface of the tissue on agar was analyzed identically to pure cultures grown on agar. Identical trends between pure bacteria cultures and stamps from tissue surface verified that the inoculated bacteria did grow on the tissue surface as expected (Fig. 7).

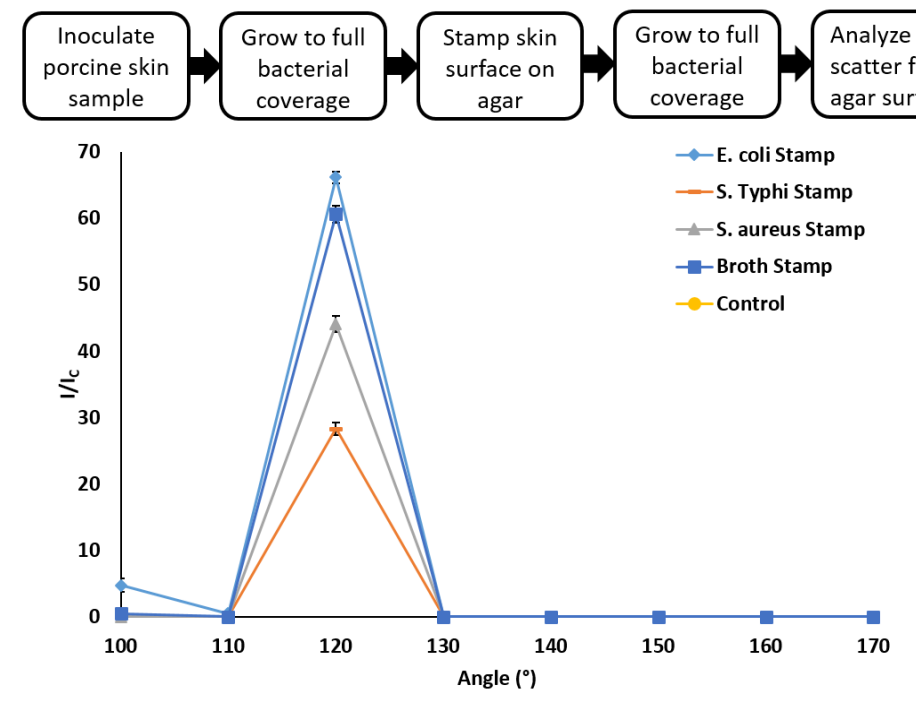

Figure 7. Mie scatter spectra from porcine skin stamps on agar. Following bacterial inoculation and growth on the exposed dermis of porcine skin samples, the skin surface was stamped onto LB-Miller agar and grown to full coverage of the agar surface. Mie scatter from the agar surface was analyzed and normalized identically to pure bacteria cultures on agar. In each case pictures of the agar analyzed are shown, which indicate that there is very little difference that can be detected visually despite differences in Mie scatter.

\subsection{Translation to clinical use}

Unique scatter spectra are detected on a porcine skin model and on human cadaveric skin. Through the use of human cadaveric skin, we have shown that our device is capable of detection of pathogenic bacteria in a human model, and 
therefore should have the same outcome in a clinical setting. The photodiode array designed in this study could then be modified to simply scan a wound on the body to analyze the presence of an infection in real time. The data in this study was acquired in three seconds, so an immediate analysis could be performed. Translation to a smartphone application would allow for immediate and non-destructive diagnosis of wound infection in a harmless, painless, and contact-free manner at regular time points.

\subsection{Future impact}

The angular photodiode array designed could act as a standalone device or smartphone attachment, with the LED flash filtered to $650 \mathrm{~nm}$ to act as the light source. Unlike the previous studies discussed above ${ }^{22,23,24}$, where NIR or IR was used as a light source, a simple red color was utilized in this study, potentially enabling the use of smartphone as both a light source and a light detector, as well as providing reduced noise, stronger signal, and less signal conditioning. Converting this technology to be used with a smartphone would allow for remote diagnosis of a bacterial infection anywhere that a smartphone could be accessed. With the technology developed through this work, patients can monitor infection at home and be alerted to seek medical attention as soon as an infection begins. The developed technology could be used in areas where immediate access to a physician is not possible, so infection can be diagnosed before it is able to become limb or life threatening.

\subsection{Limitations}

Variation seen in the presented data is likely due to the presence of commensal bacteria on skin. Skin has high numbers of non-pathogenic bacteria, this system does pick up signals from these commensal bacteria. Variation due to commensal bacteria is reduced in this study by normalizing data to control samples. In a clinical setting, data could be normalized to an area of skin that is not affected by a wound to reduce variation due to natural, healthy bacteria presence.

\section{CONCLUSION}

A portable and inexpensive device was successfully designed to diagnose pathogenic bacteria presence in a wound-like environment. Species-specific detection of bacterial infections on tissue samples is possible with the angular photodiode array developed in this study. Bacterial species that are different shapes, sizes, and Gram stain show unique trends across various angles of detection. Information obtained from our device can determine if an infection is present, and, if so, the responsible bacterial species and therefore the best antibiotic treatment. Common skin contaminants have little to no impact on the Mie scatter spectra from tissue samples, which is promising for translation to clinical use.

\section{ACKNOWLEDGMENTS}

The authors appreciate Dr. Dustin K. Harshman for the design of initial photodiode array and accompanying circuitry and Prof. L. Daniel Latt for providing human cadaveric skin samples, all at the University of Arizona. This work was supported by Cardiovascular Biomedical Engineering Training Grant from U.S. National Institutes of Health (NIH), grant number T32HL007955. The preliminary results of this work have been filed as a U.S. patent application number $62 / 320,093$.

\section{REFERENCES}

[1] Dargaville, T.R., Farrugia, B.L., Broadbent, J.A., Pace, S., Upton, Z., Voelcker, N.H., "Sensors and imaging for wound healing: A review," Biosens. Bioelectron. 41, 30-42 (2013).

[2] Lipsky, B.A., Berendt, A.R., Cornia, P.B., Pile, J.C., Peters, E.J.G., Armstrong, D.G., Deery, H.G., Embil, J.M., Joseph, W.S., Karchmer, A.W., Pinzur, M.S., Senneville, E., "2012 infectious diseases Society of America Clinical Practice guideline for the diagnosis and treatment of diabetic foot infections," Clin. Infect. Dis. 54, e132-e173 (2012).

[3] Stevens, D.L., Bisno, A.L., Chambers, H.F., Dellinger, E.P., Goldstein, E.J.C., Gorbach, S.L., Hirchmann, J.V., Kaplan, S.L., Montoya, J.G., Wade, J.C., "Practice guidelines for the diagnosis and management of skin and soft tissue infections: 2014 update by the Infectious Diseases Society of America," Clin. Infect. Dis. 59, e10-e52 (2014). 
[4] Markova, A., Mostow, E.N., "US skin disease assessment: ulcer and wound care," Dermatol. Clin. 30, 107-111 (2012).

[5] Sen, C.K., Gordilla, G.M., Roy, S., Kirsner, R., Lambert, L., Hunt, T.K., Gottrup, F., Gurtner, G.C., Longtaker, M.T., "Human skin wounds: A major and snowballing threat to public health and the economy," Wound Rep. Reg. 17, 763-771 (2009).

[6] Rondas, A.A.L.M., Schols, J.M.G.A., Halfens, R.J.G., Stobberingh, E.E., "Swab versus biopsy for the diagnosis of chronic infected wounds," Adv. Skin Wound Care 26, 211-219 (2013).

[7] Reddy, M., Gill, S.S., Wu, W., Kalkar, S.R., Rochon, P.A., "Does this patient have an infection of a chronic wound?," JAMA 307, 605-611 (2012).

[8] CDC (U.S. Center for Disease Control and Prevention), 2014. National Diabetes Statistics Report: Estimates of Diabetes and Its Burden in the United States, 2014. CDC, Atlanta.

[9] Pendsey, S.P., “Understanding diabetic foot,” Int. J. Diabetes Dev. Ctries. 30, $75-79$ (2010).

[10] Pickwell, K., Siersma, V., Kars, M., Apelqvist, J., Bakker, K., Edmonds, M., Holstein, P., Jirkovska, A., Jude, E., Mauricio, D., Piaggesi, A., Tennvall, G.R., Reike, H., Spraul, M., Ucciolo, L., Urbancic, V., van Acker, K., van Baal, J., Schaper, N., "Predictors of lower-extremity amputation in patients with an infected diabetic foot ulcer," Diabetes Care 38, 852-857 (2015).

[11]Frykberg, R.G., Marston, W.A., Cardinal, M., "The incidence of lower-extremity amputation and bone resection in diabetic foot ulcer patients treated with a human fibroblast-derived dermal substitute," Adv. Skin Wound Care 28, 17-20 (2015).

[12] Brubaker, A.L., Rendon, J.L., Ramirez, L., Choudhry, M.A., Kovacs, E.J., "Reduced neutrophil chemotaxis and infiltration contributes to delayed resolution of cutaneous wound infection with advanced age," J. Immunol. 190, 1746-1757 (2013).

[13] Azzopardi, E.A., Azzopardi, E., Camilleri, L., Villapalos, J., Boyce, D.E., Dziewulski, P., Dickson, W.A., Whitaker, I.S., "Gram Negative Wound Infection in Hospitalized Adult Burn Patients-Systematic Review and Metanalysis-," PloS ONE 9, e95042 (2014).

[14] Fournier, A., Pantet, O., Guerid, S., Eggimann, P., Pagani, J.L., Revelly, J.P., Hauser, P.M., Marchetti, O., Fontanella, S., Letovanec, I., Ravat, F., Berger, M.M., Pannatier, A., Voirol, P., Que, Y.A., "Effective treatment of invasive Aspergillus fumigatus infection using combination of topical and systemic antifungals in severely burned patient," J. Burn Care Res. 36, e85-e89 (2015).

[15] Korol, E., Johnston, K., Waser, N., Sifakis, F., Jafri, H.S., Lo, M., Kyaw, M.H., “A systematic review of risk factors associated with surgical site infection among surgical patients," PLoS ONE 8, e83743 (2013).

[16] Ortega, G., Rhee, D.S., Papandria, D.J., Yang, J., Ibrahim, A.M., Shore, A.D., Makary, M.A., Abdullah, F., “An evaluation of surgical site infections by wound classification system using the ACS-NSQIP," J Surg. Res. 174, 3338 (2012).

[17]Zehtabchi, S., Tan, A., Yadav, K., Badawy, A., Lucchesi, M., "The impact of wound age on the infection rate of simple lacerations repaired in the emergency department," Injury Int. J. Care Injured 43, 1793-1798 (2012).

[18] Liang, P.S., Park, T.S., Yoon, J.Y., "Rapid and reagentless detection of microbial contamination within meat utilizing a smartphone-based biosensor," Sci. Rep. 4, 5953 (2014).

[19] Park, T.S., Li, W., McCracken, K.E., Yoon, J.Y., "Smartphone quantifies Salmonella from paper microfluidics," Lab Chip 13, 4832-4840 (2013).

[20] Cho, S., Park, T.S., Nahapetian, T.G., Yoon, J.Y., "Smartphone-based, sensitive $\mu$ PAD detection of urinary tract infection and Gonorrhea," Biosens. Bioelecton. 74, 601-611 (2015).

[21] Liang, P.S., Park, T.S., Yoon, J.Y., Light scattering based detection of food pathogens, in: Lu, R. (Ed.), Light Scattering Technology for Food Property, Quality and Safety Assessment. Taylor \& Francis: Abingdon, 429-444 (2016).

[22] Banada, P.P., Huff, K., Bae, E., Rajwa, B., Aroonnual, A., Bayraktar, B., Adil, A., Robinson, J.P., Hirleman, E.D., Bhunia, A.K, "Label-free detection of multiple bacterial pathogens using light-scattering sensor," Biosens. Bioelecton. 24, 1685-1692 (2009).

[23] Singh, A.K., Bettasso, A.M., Bae, E., Rajwa, B., Dundar, M.M., Forster, M.D., Liu, L., Barrett, B., Lovchik, J., Robinson, J.P., Hirleman, E.D., Bhunia, A.K., "Laser optical sensor, a label-free on-plate Salmonella enterica colony detection tool," mBio 5, e01019-13 (2014).

[24] Tang, Y., Kim, H., Singh, A.K., Aroonnual, A., Bae, E., Rajwa, B., Fratamico, P.M., Bhunia, A.K., "Light scattering sensor for direct identification of colonies of Escherichia coli serogroups O26, O45, O103, O111, O121, O145 and O157," PLoS ONE 9, e105272 (2014). 